\title{
A Mixed Finite Element Method for the Biharmonic Problem Using Biorthogonal or Quasi-Biorthogonal Systems
}

\author{
Bishnu P. Lamichhane
}

Received: 22 February 2009 / Accepted: 20 July 2010 / Published online: 3 August 2010

(C) Springer Science+Business Media, LLC 2010

\begin{abstract}
We consider a finite element method based on biorthogonal or quasi-biorthogonal systems for the biharmonic problem. The method is based on the primal mixed finite element method due to Ciarlet and Raviart for the biharmonic equation. Using different finite element spaces for the stream function and vorticity, this approach leads to a formulation only based on the stream function. We prove optimal a priori estimates for both stream function and vorticity, and present numerical results to demonstrate the efficiency of the approach.
\end{abstract}

Keywords Biharmonic problem · Mixed finite elements · Biorthogonal or quasi-biorthogonal system

\section{Introduction}

Fourth order elliptic and parabolic problems appear in many applications. Some examples are thin beams and plates, strain gradient elasticity [8, 12], the Stokes problem [16] and phase separation of a binary mixture [31]. The variational formulation of these problems requires $H^{2}$-conforming finite elements, which are not so easy to construct in unstructured meshes. This difficulty can be avoided either by using a discontinuous Galerkin method as in $[6,12,31]$ or by using a mixed formulation as in $[3,8-11,13,14,26]$.

In this paper, we analyze the primal mixed finite method due to Ciarlet and Raviart [8-10] using different spaces for the stream function and vorticity. We work with discrete spaces having local basis functions satisfying the condition of biorthogonality or quasibiorthogonality for the discretization of the stream function and vorticity. The condition of biorthogonality or quasi-biorthogonality is essential in our construction as it allows us to

B.P. Lamichhane

School of Mathematical \& Physical Sciences, University of Newcastle, University Drive, NSW 2308, Callaghan, Australia

B.P. Lamichhane $(\bowtie)$

Mathematical Sciences Institute, Australian National University, ACT 0200, Canberra, Australia

e-mail: Bishnu.Lamichhane@newcastle.edu.au 
statically condense out the degree of freedom associated with the vorticity and the Lagrange multiplier and arrive at a formulation involving only the degree of freedom associated with the stream function. Hence a reduced system is obtained, which is easy to solve. The vorticity and the Lagrange multiplier can be computed in a post-processing step.

The structure of the rest of the paper is organized as follows. In the next section, we briefly recall a mixed formulation for the biharmonic equation suitable for our analysis. Section 3 is devoted for the numerical analysis of the approach. We also present the algebraic formulation of the problem and briefly discuss how the static condensation can be applied to get a formulation only based on the stream function. Finally, we show some numerical experiments in Sect. 4.

\section{A Mixed Formulation of Biharmonic Equation}

In this section we briefly recall a mixed formulation of the biharmonic problem. Let $\Omega$ in $\mathbb{R}^{2}$ be a bounded convex domain with polygonal boundary $\partial \Omega$ and outward pointing normal $\boldsymbol{n}$ on $\partial \Omega$. The biharmonic equation

$$
\Delta^{2} u=f \quad \text { in } \Omega
$$

with simply supported boundary condition

$$
u=\Delta u=0 \quad \text { on } \partial \Omega,
$$

or with clamped boundary condition

$$
u=\frac{\partial u}{\partial \boldsymbol{n}}=0 \quad \text { on } \partial \Omega
$$

is studied extensively in $[3,5,6,8-10,13,14,26,31]$.

Here we consider a new discretization scheme of the mixed formulation presented in [8-10], where the variational formulation is based on the stream function, the vorticity and the Lagrange multiplier. The central idea of our approach is to use the sets of basis functions which form a biorthogonal or quasi-biorthogonal system for approximating the stream function and vorticity.

We introduce some notations for the subsequent use. Let $u: \Omega \rightarrow \mathbb{R}$ be a real-valued function. For $1 \leq p<\infty$, the Lebesgue space $L^{p}(\Omega)$ is defined as

$$
L^{p}(\Omega):=\left\{\left.u\left|\int_{\Omega}\right| u(x)\right|^{p} d x<\infty\right\}
$$

with a natural norm defined by $\|u\|_{L^{p}(\Omega)}:=\left(\int_{\Omega}|u(x)|^{p} d x\right)^{1 / p}$, and for $p=\infty$

$$
L^{\infty}(\Omega):=\{u \mid \text { ess } \sup \{|u(x)|, x \in \Omega\}<\infty\}
$$

with a norm $\|u\|_{L^{\infty}(\Omega)}:=\operatorname{ess} \sup \{|u(x)|, x \in \Omega\}$.

Denoting the distributional derivative by $D$ and a multi-index by $\alpha \in \mathbb{N}_{0}^{2}$, for a nonnegative integer $s$, a Sobolev space $W^{s, p}(\Omega)$ for $p \in[1, \infty]$ is the set of all functions $u \in$ $L^{p}(\Omega)$ such that for each multi-index $\alpha \in \mathbb{N}_{0}^{2}$ with $|\alpha| \leq s, D^{\alpha} u$ exists and $D^{\alpha} u \in L^{p}(\Omega)$. We recall that $|\alpha|=\alpha_{1}+\alpha_{2}$. The norm in the space $W^{s, p}(\Omega)$ is defined as

$$
\|u\|_{s, p, \Omega}:= \begin{cases}\left(\sum_{|\alpha| \leq s}\left\|D^{\alpha} u\right\|_{L^{p}(\Omega)}^{p}\right)^{1 / p}, & 1 \leq p<\infty \\ \max _{|\alpha| \leq s}\left\|D^{\alpha} u\right\|_{L^{\infty}(\Omega)}, & p=\infty .\end{cases}
$$


When $p=2$, the Sobolev spaces $L^{p}(\Omega)$ and $W^{s, p}(\Omega)$ are Hilbert spaces and inner products can be defined for them which induce the norm as defined above. The inner product for the space $L^{2}(\Omega)$ of all square-integrable functions in $\Omega$ is defined as $(u, v)_{0, \Omega}:=\int_{\Omega} u v d x$, and that of the space $W^{s, 2}(\Omega)$ is defined as

$$
(u, v)_{s, \Omega}:=\left(\sum_{|\alpha| \leq s} D^{\alpha} u, \sum_{|\alpha| \leq s} D^{\alpha} v\right)_{0, \Omega} .
$$

For the purpose of simplicity, the norm in the space $L^{2}(\Omega)$ is then denoted by $\|\cdot\|_{0, \Omega}$, and that of the space $W^{s, 2}(\Omega)$ is denoted by $\|\cdot\|_{s, \Omega}$. It is standard to write $H^{s}(\Omega)$ for the space $W^{s, p}(\Omega)$ when $p=2$. The space $H_{0}^{s}(\Omega)$ is defined by completing the space $C_{0}^{\infty}(\Omega)$ with respect to the norm $\|\cdot\|_{s, \Omega}$. The semi-norm on the space $H^{s}(\Omega)$ or $H_{0}^{s}(\Omega)$ is given by

$$
|u|_{s, \Omega}=\sum_{|\alpha|=s}\left\|D^{\alpha} u\right\|_{0, \Omega}
$$

Sobolev spaces $H^{s}(\Omega)$ for real $s>0$ can be defined by using real interpolation [24]. We refer to $[1,17,24]$ for more information on Sobolev spaces.

Let $H_{B}^{2}(\Omega)$ stands for $H_{0}^{2}(\Omega)$ for the clamped boundary case and for $H^{2}(\Omega) \cap H_{0}^{1}(\Omega)$ for the simply supported case. We consider the following variational form of the biharmonic problem

$$
J(u)=\inf _{v \in H_{B}^{2}(\Omega)} J(v)
$$

with

$$
J(v)=\frac{1}{2} \int_{\Omega}|\Delta v|^{2} d x-\int_{\Omega} f v d x .
$$

To write the weak or variational formulation of the boundary value problem, we introduce the space $\boldsymbol{V}=H_{0}^{1}(\Omega) \times L^{2}(\Omega)$ of the stream function and vorticity with the inner product $(\cdot, \cdot)_{\boldsymbol{V}}$ defined as

$$
((u, \phi),(v, \psi))_{\boldsymbol{V}}=(\nabla u, \nabla v)_{0, \Omega}+(\phi, \psi)_{0, \Omega}
$$

and with the norm $\|\cdot\|_{\boldsymbol{V}}$ induced by this inner product. Let $Q=H_{0}^{1}(\Omega)$ for the simply supported case and $Q=H^{1}(\Omega)$ for the clamped case. The variational problem (2.4) can be recast as the minimization problem [8]

$$
\mathcal{J}(u, \phi)=\inf _{(v, \psi) \in \mathcal{V}} \mathcal{J}(v, \psi),
$$

where

$$
\begin{aligned}
\mathcal{J}(v, \psi) & =\frac{1}{2} \int_{\Omega}|\psi|^{2} d x-\int_{\Omega} f v d x, \\
\mathcal{V} & =\left\{(v, \psi) \in \boldsymbol{V}: \int_{\Omega} \nabla v \cdot \nabla q+\psi q d x=0, q \in Q\right\} .
\end{aligned}
$$

The problem (2.6) can be recast as a saddle point formulation: given $\ell \in H^{-1}(\Omega)$ find $((u, \phi), p) \in \boldsymbol{V} \times Q$ so that

$$
\begin{aligned}
a((u, \phi),(v, \psi))+b((v, \psi), p) & =\ell(v), \quad(v, \psi) \in \boldsymbol{V}, \\
b((u, \phi), q) & =0, \quad q \in Q,
\end{aligned}
$$


where

$$
\begin{aligned}
a((u, \phi),(v, \psi)) & =\int_{\Omega} \phi \psi d x, b((v, \psi), q)=\int_{\Omega} \nabla v \cdot \nabla q+\psi q d x, \\
\ell(v) & =\int_{\Omega} f v d x .
\end{aligned}
$$

This mixed formulation is introduced in $[8,10]$, where the existence and uniqueness of the solution is also established, see also [9]. A method based on this mixed formulation leading to an unconstrained approach using a dual mesh is proposed in [11] for the linear finite elements.

\section{Finite Element Discretizations}

We consider a quasi-uniform and shape-regular triangulation $\mathcal{T}_{h}$ of the polygonal domain $\Omega$, where $\mathcal{T}_{h}$ consists of triangles or parallelograms. Let $S_{h} \subset H^{1}(\Omega)$ be a standard Lagrange finite element space, $S_{h}^{0}=S_{h} \cap H_{0}^{1}(\Omega)$, and $M_{h} \subset L^{2}(\Omega)$ be another finite element space. Let $\boldsymbol{V}_{h}=S_{h}^{0} \times M_{h}, Q_{h}=S_{h}^{0}$ for the simply supported case, $Q_{h}=S_{h}$ for the clamped case, and $\operatorname{dim} M_{h}=\operatorname{dim} Q_{h}$ for both cases. In the following, we use a generic constant $C$, which takes different values in different occurrences but is always independent of the mesh-size.

We assume that there is a constant $\beta>0$ independent of the mesh-size such that

$$
\left\|\mu_{h}\right\|_{0, \Omega} \leq \beta \sup _{q_{h} \in Q_{h} \backslash\{0\}} \frac{\int_{\Omega} q_{h} \mu_{h} d x}{\left\|q_{h}\right\|_{0, \Omega}}, \quad \mu_{h} \in M_{h},
$$

and the space $M_{h}$ has the approximation property:

$$
\inf _{\lambda_{h} \in M_{h}}\left\|\phi-\lambda_{h}\right\|_{0, \Omega} \leq C h^{k}|\phi|_{k, \Omega}, \quad \phi \in H^{k}(\Omega) .
$$

Moreover, the spaces $S_{h}$ and $S_{h}^{0}$ are assumed to have the following approximation properties:

$$
\begin{array}{ll}
\inf _{v_{h} \in S_{h}}\left\|u-v_{h}\right\|_{1, \Omega} \leq C h^{k}|u|_{k+1, \Omega}, & u \in H^{k+1}(\Omega), \\
\inf _{v_{h} \in S_{h}^{0}}\left\|u-v_{h}\right\|_{1, \Omega} \leq C h^{k}|u|_{k+1, \Omega}, & u \in H^{k+1}(\Omega) \cap H_{0}^{1}(\Omega) .
\end{array}
$$

The discrete biharmonic problem is then given by: given $\ell \in H^{-1}(\Omega)$, find $\left(\left(u_{h}, \phi_{h}\right), p_{h}\right) \in$ $V_{h} \times Q_{h}$ such that

$$
\begin{gathered}
a\left(\left(u_{h}, \phi_{h}\right),\left(v_{h}, \psi_{h}\right)\right)+b\left(\left(v_{h}, \psi_{h}\right), p_{h}\right)=\ell\left(v_{h}\right), \quad\left(v_{h}, \psi_{h}\right) \in V_{h}, \\
b\left(\left(u_{h}, \phi_{h}\right), q_{h}\right)=0, \quad q_{h} \in Q_{h} .
\end{gathered}
$$

The kernels of the bilinear form $b(\cdot, \cdot)$ for the continuous and discrete setting are denoted by $\operatorname{Ker} B$ and $\operatorname{Ker} B_{h}$, respectively. We note that

$\operatorname{Ker} B=\mathcal{V} \quad$ and $\quad \operatorname{Ker} B_{h}=\left\{\left(v_{h}, \psi_{h}\right) \in V_{h}: \int_{\Omega} \nabla v_{h} \cdot \nabla q_{h}+\psi_{h} q_{h} d x=0, q_{h} \in Q_{h}\right\}$.

Following Ciarlet [8], we show the coercivity of the bilinear form $a(\cdot, \cdot)$ on $\operatorname{Ker} B_{h}$. 
Lemma 3.1 There exists $\alpha_{0}>0$ such that

$$
a\left(\left(v_{h}, \psi_{h}\right),\left(v_{h}, \psi_{h}\right)\right) \geq \alpha_{0}\left(\left|v_{h}\right|_{1, \Omega}^{2}+\left\|\psi_{h}\right\|_{0, \Omega}^{2}\right), \quad\left(v_{h}, \psi_{h}\right) \in \operatorname{Ker} B_{h} .
$$

Proof As $\left(v_{h}, \psi_{h}\right) \in \operatorname{Ker} B_{h}$, the second equation of (3.4) yields

$$
\int_{\Omega} \nabla v_{h} \cdot \nabla q_{h}+\psi_{h} q_{h} d x=0, \quad q_{h} \in Q_{h}
$$

Choosing $q_{h}=v_{h}$ in the above equation, we have

$$
\int_{\Omega} \nabla v_{h} \cdot \nabla v_{h}+\psi_{h} v_{h} d x=0
$$

and hence

$$
\left|v_{h}\right|_{1, \Omega}^{2}+\int_{\Omega} \psi_{h} v_{h} d x=0
$$

Using Cauchy-Schwarz and Poincaré inequality, we obtain

$$
\left|v_{h}\right|_{1, \Omega}^{2} \leq C\left|v_{h}\right|_{1, \Omega}\left\|\psi_{h}\right\|_{0, \Omega},
$$

which gives

$$
\left|v_{h}\right|_{1, \Omega} \leq C\left\|\psi_{h}\right\|_{0, \Omega}
$$

Hence

$$
a\left(\left(v_{h}, \psi_{h}\right),\left(v_{h}, \psi_{h}\right)\right)=\left\|\psi_{h}\right\|_{0, \Omega}^{2} \geq \frac{1}{C^{2}}\left|v_{h}\right|_{1, \Omega}^{2} .
$$

Summing this inequality with $a\left(\left(v_{h}, \psi_{h}\right),\left(v_{h}, \psi_{h}\right)\right)=\left\|\psi_{h}\right\|_{0, \Omega}^{2}$ and dividing by two, we obtain the desired result with $2 \alpha_{0}=\min \left(\frac{1}{C^{2}}, 1\right)$.

Lemma 3.2 Let $((u, \phi), p) \in \boldsymbol{V} \times Q$ be the solution of the continuous problem (2.9) with $p \in H^{k}(\Omega)$ and $\left(\left(u_{h}, \phi_{h}\right), p_{h}\right) \in V_{h} \times Q_{h}$ be that of discrete problem (3.4). Then there exists a constant $C>0$ independent of the mesh-size $h$ so that

$$
\left\|\left(u-u_{h}, \phi-\phi_{h}\right)\right\| \boldsymbol{V} \leq C\left(\inf _{\left(w_{h}, \xi_{h}\right) \in \operatorname{Ker} B_{h}}\left\|\left(u-w_{h}, \phi-\xi_{h}\right)\right\| V^{k}+h^{k}|p|_{k, \Omega}\right) .
$$

Proof Let $\left(w_{h}, \xi_{h}\right) \in \operatorname{Ker} B_{h}$ so that $\left(u_{h}-w_{h}, \phi_{h}-\xi_{h}\right) \in \operatorname{Ker} B_{h}$, and hence coercivity of $a(\cdot, \cdot)$ on $\operatorname{Ker} B_{h}$ yields

$$
\alpha_{0}\left\|\left(u_{h}-w_{h}, \phi_{h}-\xi_{h}\right)\right\|_{\boldsymbol{V}} \leq \sup _{\left(v_{h}, \psi_{h}\right) \in \operatorname{Ker} B_{h}} \frac{a\left(\left(u_{h}-w_{h}, \phi_{h}-\xi_{h}\right),\left(v_{h}, \psi_{h}\right)\right)}{\left\|\left(v_{h}, \psi_{h}\right)\right\|_{\boldsymbol{V}}} .
$$

Since $a\left(\left(u-u_{h}, \phi-\phi_{h}\right),\left(v_{h}, \psi_{h}\right)\right)+b\left(\left(v_{h}, \psi_{h}\right), p\right)=0$ for all $\left(v_{h}, \psi_{h}\right) \in \operatorname{Ker} B_{h}$, we have

$$
\begin{aligned}
a\left(\left(u_{h}-w_{h}, \phi_{h}-\xi_{h}\right),\left(v_{h}, \psi_{h}\right)\right)= & a\left(\left(u-w_{h}, \phi-\xi_{h}\right),\left(v_{h}, \psi_{h}\right)\right) \\
& +a\left(\left(u_{h}-u, \phi_{h}-\phi\right),\left(v_{h}, \psi_{h}\right)\right) \\
= & a\left(\left(u-w_{h}, \phi-\xi_{h}\right),\left(v_{h}, \psi_{h}\right)\right)+b\left(\left(v_{h}, \psi_{h}\right), p\right) .
\end{aligned}
$$


Denoting the projection of $p$ onto $Q_{h}$ with respect to $H^{1}$-inner product by $\tilde{p}_{h}$, we have

$$
\int_{\Omega} \nabla v_{h} \cdot \nabla\left(p-\tilde{p}_{h}\right) d x=-\int_{\Omega} v_{h}\left(p-\tilde{p}_{h}\right) d x .
$$

As $\left(v_{h}, \psi_{h}\right) \in \operatorname{Ker} B_{h}$, using (3.8),

$$
b\left(\left(v_{h}, \psi_{h}\right), p\right)=b\left(\left(v_{h}, \psi_{h}\right), p-\tilde{p}_{h}\right)=-\int_{\Omega} v_{h}\left(p-\tilde{p}_{h}\right) d x+\int_{\Omega} \psi_{h}\left(p-\tilde{p}_{h}\right) d x,
$$

and hence approximation property of $\tilde{p}_{h}$ yields

$$
\left|b\left(\left(v_{h}, \psi_{h}\right), p\right)\right| \leq C h^{k}|p|_{k, \Omega}\left\|\left(v_{h}, \psi_{h}\right)\right\| \boldsymbol{V} .
$$

Thus

$$
\begin{aligned}
\alpha_{0}\left\|\left(u_{h}-w_{h}, \phi_{h}-\xi_{h}\right)\right\|_{\boldsymbol{V}} & \leq \sup _{\left(v_{h}, \psi_{h}\right) \in \operatorname{Ker} B_{h}} \frac{a\left(\left(u-w_{h}, \phi-\xi_{h}\right),\left(v_{h}, \psi_{h}\right)\right)}{\left\|\left(v_{h}, \psi_{h}\right)\right\| \boldsymbol{V}}+C h^{k}|p|_{k, \Omega} \\
& \leq\|a\|\left\|\left(u-w_{h}, \phi-\xi_{h}\right)\right\| \boldsymbol{V}+C h^{k}|p|_{k, \Omega} .
\end{aligned}
$$

Finally, a triangle inequality yields the estimate (3.7):

$$
\begin{aligned}
\left\|\left(u-u_{h}, \phi-\phi_{h}\right)\right\|_{\boldsymbol{V}} & \leq\left\|\left(u-w_{h}, \phi-\xi_{h}\right)\right\|_{\boldsymbol{V}}+\left\|\left(w_{h}-u_{h}, \xi_{h}-\phi_{h}\right)\right\|_{\boldsymbol{V}} \\
& \leq\left(1+\frac{\|a\|}{\alpha_{0}}\right)\left\|\left(u-w_{h}, \phi-\xi_{h}\right)\right\|_{\boldsymbol{V}}+\frac{C}{\alpha_{0}} h^{k}|p|_{k, \Omega} .
\end{aligned}
$$

Lemma 3.3 Let us assume the simply supported boundary condition $\left(Q=H_{0}^{1}(\Omega)\right.$ and $\left.Q_{h}=S_{h}^{0}\right)$. Let $\left(w_{h}, \xi_{h}\right) \in \operatorname{Ker} B_{h},(w, \xi) \in \operatorname{Ker} B$, and $R_{h}: H^{1}(\Omega) \rightarrow S_{h}^{0}$ be the Ritzprojector defined as

$$
\int_{\Omega} \nabla\left(R_{h} w-w\right) \cdot \nabla v_{h} d x=0, \quad v_{h} \in S_{h}^{0}
$$

Then

$$
\left|w-w_{h}\right|_{1, \Omega} \leq\left\|\xi-\xi_{h}\right\|_{0, \Omega}+\left|R_{h} w-w\right|_{1, \Omega}
$$

Proof The idea of the proof is similar to Proposition 3 in [11]. Since $\left(w_{h}, \xi_{h}\right) \in \operatorname{Ker} B_{h}$ and $(w, \xi) \in \operatorname{Ker} B$ satisfy

$$
\begin{aligned}
\int_{\Omega} \nabla w \cdot \nabla q+\xi q d x & =0, \quad q \in Q, \quad \text { and } \\
\int_{\Omega} \nabla w_{h} \cdot \nabla q_{h}+\xi_{h} q_{h} d x & =0, \quad q_{h} \in Q_{h},
\end{aligned}
$$

respectively, we have

$$
\int_{\Omega} \nabla\left(w-w_{h}\right) \cdot \nabla q_{h}+\left(\xi-\xi_{h}\right) q_{h} d x=0, \quad q_{h} \in Q_{h} .
$$


Using the Ritz-projector $R_{h}$, we can rewrite (3.9) as

$$
\int_{\Omega} \nabla\left(R_{h} w-w_{h}\right) \cdot \nabla q_{h}+\left(\xi-\xi_{h}\right) q_{h} d x=0, \quad q_{h} \in Q_{h} .
$$

Taking $q_{h}=R_{h} w-w_{h}$ in equation (3.10) and using Cauchy-Schwarz and Poincaré inequality, we obtain

$$
\begin{aligned}
\left|R_{h} w-w_{h}\right|_{1, \Omega}^{2} & \leq\left\|\xi-\xi_{h}\right\|_{0, \Omega}\left\|R_{h} w-w_{h}\right\|_{0, \Omega} \\
& \leq C\left\|\xi-\xi_{h}\right\|_{0, \Omega}\left|R_{h} w-w_{h}\right|_{1, \Omega}
\end{aligned}
$$

which yields

$$
\left|R_{h} w-w_{h}\right|_{1, \Omega} \leq C\left\|\xi-\xi_{h}\right\|_{0, \Omega} .
$$

A triangle inequality gives the final result:

$$
\left|w-w_{h}\right|_{1, \Omega} \leq\left|R_{h} w-w_{h}\right|_{1, \Omega}+\left|w-R_{h} w\right|_{1, \Omega} \leq C\left\|\xi-\xi_{h}\right\|_{0, \Omega}+\left|w-R_{h} w\right|_{1, \Omega} .
$$

In order to analyze the approximation property of $\phi_{h}$, we introduce a quasi-projection operator: $\Pi_{h}: L^{2}(\Omega) \rightarrow M_{h}$, which is defined as

$$
\int_{\Omega} \Pi_{h} v q_{h} d x=\int_{\Omega} v q_{h} d x, v \in L^{2}(\Omega), \quad q_{h} \in Q_{h} .
$$

It is easy to verify that $\Pi_{h}$ is well-defined due to assumption (3.1) and $\operatorname{dim} M_{h}=\operatorname{dim} Q_{h}$, and is identity if restricted to $M_{h}$. Hence $\Pi_{h}$ is a projection onto the space $M_{h}$. We note that $\Pi_{h}$ is not the orthogonal projection onto $M_{h}$ but an oblique projection onto it. Oblique projectors are studied extensively in [15], and different proofs on an identity on the norm of oblique projections are provided in [30]. This type of operator is introduced in [28] in the context of interpolation of non-smooth functions, and is extensively used in the context of mortar finite elements $[18,19,32]$. It is useful to note that $\Pi_{h}$ is stable in $L^{2}$ norm $[4,18]$. Furthermore, the approximation property (3.2) of the space $M_{h}$ and the projection property of the operator $\Pi_{h}$ gives the following lemma.

Lemma 3.4 Under assumptions (3.1) and (3.2), there exists a constant $C>0$ independent of the mesh-size $h$ so that

$$
\left\|v-\Pi_{h} v\right\|_{0, \Omega} \leq C h^{r}|v|_{r, \Omega}, \quad v \in H^{s}(\Omega), s \geq 0,
$$

where $r=\min (s, k)$.

Theorem 3.5 Let $((u, \phi), p) \in \boldsymbol{V} \times Q$ be the solution of the continuous problem (2.9) and $\left(\left(u_{h}, \phi_{h}\right), p_{h}\right) \in V_{h} \times Q_{h}$ be that of discrete one (3.4) for the simply supported case $\left(Q=H_{0}^{1}(\Omega)\right.$ and $\left.Q_{h}=S_{h}^{0}\right)$. Let $u \in H^{k+1}(\Omega) \cap H_{0}^{1}(\Omega), \phi \in H^{k}(\Omega) \cap H_{0}^{1}(\Omega), p \in H^{k}(\Omega)$, and assumptions (3.1), (3.2) and (3.3) are satisfied. Then there exists a constant $C>0$ independent of the mesh-size $h$ so that

$$
\left\|\left(u-u_{h}, \phi-\phi_{h}\right)\right\|_{\boldsymbol{V}} \leq C h^{k}\left(|u|_{k+1, \Omega}+|\phi|_{k, \Omega}+|p|_{k, \Omega}\right) .
$$


Proof Let $\left(w_{h}, \xi_{h}\right) \in \boldsymbol{V}_{h}$ be defined as

$$
\begin{array}{rlrl}
\int_{\Omega}\left(\phi-\xi_{h}\right) q_{h} d x & =0, \quad q_{h} \in Q_{h}, \quad \text { and } \\
\int_{\Omega} \nabla w_{h} \nabla q_{h}+\xi_{h} q_{h} d x & =0, \quad q_{h} \in Q_{h} . &
\end{array}
$$

Hence $\left(w_{h}, \xi_{h}\right) \in \operatorname{Ker} B_{h}$ with $\xi_{h}=\Pi_{h} \phi$, and it is easy to see that such an element is uniquely defined. Lemma 3.4 ensures that

$$
\left\|\phi-\xi_{h}\right\|_{0, \Omega} \leq C h^{k}|\phi|_{k, \Omega}
$$

Moreover, Ritz projector $R_{h}$ as defined in Lemma 3.3 has the approximation property

$$
\left|u-R_{h} u\right|_{1, \Omega} \leq C h^{k}|u|_{k+1, \Omega} .
$$

Hence using the result of Lemma 3.3 we obtain

$$
\left|u-w_{h}\right|_{1, \Omega} \leq\left\|\phi-\xi_{h}\right\|_{0, \Omega}+\left|u-R_{h} u\right|_{1, \Omega} \leq C h^{k}\left(|u|_{k+1, \Omega}+|\phi|_{k, \Omega}\right) .
$$

We now turn our attention to prove optimal a priori estimates in case of clamped boundary condition. For that purpose, we need a lemma. The lemma can be proved by using the ideas in [27]. See also [16, Chap. III].

Lemma 3.6 Let $k \in \mathbb{N}$ and $p \in \mathbb{R}$ such that $k \geq 1$ and $2 \leq p \leq \infty$. Let $R_{h}$ be the Ritz projection as defined in Lemma 3.3. Then for all functions $w \in W^{k+1, p}(\Omega) \cap H_{0}^{1}(\Omega)$, there exists a constant $C>0$ such that

$$
\sup _{v_{h} \in S_{h}} \frac{\int_{\Omega} \nabla\left(w-R_{h} w\right) \cdot \nabla v_{h} d x}{\left\|v_{h}\right\|_{0, \Omega}} \leq C h^{k-\frac{1}{2}-\frac{1}{p}}\|w\|_{k+1, p, \Omega} .
$$

Theorem 3.7 Let $((u, \phi), p) \in \boldsymbol{V} \times Q$ be the solution of the continuous problem (2.9) and $\left(\left(u_{h}, \phi_{h}\right), p_{h}\right) \in V_{h} \times Q_{h}$ be that of discrete one (3.4) for the clamped case $\left(Q=H^{1}(\Omega)\right.$ and $\left.Q_{h}=S_{h}\right)$. Let $u \in W^{k+1, \infty}(\Omega) \cap H_{0}^{2}(\Omega), \phi \in H^{k}(\Omega), p \in H^{k}(\Omega)$, and the assumptions (3.1), (3.2) and (3.3) are satisfied. Then there exists a constant $C>0$ independent of the mesh-size $h$ so that

$$
\left\|\left(u-u_{h}, \phi-\phi_{h}\right)\right\|_{\boldsymbol{V}} \leq C\left(h^{k}|u|_{k+1, \Omega}+h^{k}|\phi|_{k, \Omega}+h^{k-\frac{1}{2}}\|u\|_{k+1, \infty, \Omega}+h^{k}|p|_{k, \Omega}\right) .
$$

Proof Let $R_{h}$ be the Ritz projector as defined in Lemma 3.3, and $w_{h}=R_{h} u$ be the Ritz projection of $u$ onto $S_{h}^{0}$. Define $\xi_{h} \in M_{h}$ by

$$
\int_{\Omega} \nabla w_{h} \cdot \nabla q_{h}+\xi_{h} q_{h} d x=0, \quad q_{h} \in Q_{h} .
$$

Hence $\left(w_{h}, \xi_{h}\right) \in \operatorname{Ker} B_{h}$. Since $(u, \phi) \in \operatorname{Ker} B$, proceeding exactly as in Lemma 3.3, we obtain

$$
\int_{\Omega} \nabla\left(u-w_{h}\right) \cdot \nabla q_{h}+\left(\phi-\xi_{h}\right) q_{h} d x=0, \quad q_{h} \in Q_{h} .
$$


Since $\left(w_{h}, \xi_{h}\right) \in \operatorname{Ker} B_{h}$, in view of Lemma 3.2, it suffices to show that

$$
\left\|\left(u-w_{h}, \phi-\xi_{h}\right)\right\|_{\boldsymbol{V}} \leq C\left(h^{k}|u|_{k+1, \Omega}+h^{k}|\phi|_{k, \Omega}+h^{k-\frac{1}{2}}\|u\|_{k+1, \infty, \Omega}\right) .
$$

A triangle inequality allows us to write

$$
\left\|\left(u-w_{h}, \phi-\xi_{h}\right)\right\|_{\boldsymbol{V}}^{2} \leq\left|u-w_{h}\right|_{1, \Omega}^{2}+\left\|\phi-\Pi_{h} \phi\right\|_{0, \Omega}^{2}+\left\|\Pi_{h} \phi-\xi_{h}\right\|_{0, \Omega}^{2},
$$

where $\Pi_{h}$ is the quasi-projection operator defined by (3.11). It is standard that $w_{h}$ has the desired approximation property

$$
\left|u-w_{h}\right|_{1, \Omega} \leq C h^{k}|u|_{k+1, \Omega},
$$

and Lemma 3.4 ensures that

$$
\left\|\phi-\Pi_{h} \phi\right\|_{0, \Omega} \leq C h^{k}|\phi|_{k, \Omega} .
$$

Hence it is sufficient to consider the term $\left\|\Pi_{h} \phi-\xi_{h}\right\|_{0, \Omega}$ for the error estimate. We start with the stability assumption (3.1) between the spaces $M_{h}$ and $Q_{h}$ to write

$$
\begin{aligned}
\left\|\xi_{h}-\Pi_{h} \phi\right\|_{0, \Omega} & \leq C \sup _{q_{h} \in Q_{h} \backslash\{0\}} \frac{\int_{\Omega}\left(\xi_{h}-\Pi_{h} \phi\right) q_{h} d x}{\left\|q_{h}\right\|_{0, \Omega}} \\
& \leq C \sup _{q_{h} \in Q_{h} \backslash\{0\}} \frac{\int_{\Omega}\left(\xi_{h}-\phi\right) q_{h} d x}{\left\|q_{h}\right\|_{0, \Omega}} \\
& \leq C \sup _{q_{h} \in Q_{h} \backslash\{0\}} \frac{\int_{\Omega} \nabla\left(u-w_{h}\right) \cdot \nabla q_{h} d x}{\left\|q_{h}\right\|_{0, \Omega}} .
\end{aligned}
$$

Since $w_{h}$ is the Ritz projection of $u$ onto $S_{h}^{0}$, the final result follows by using Lemma 3.6 with $p=\infty$.

Remark 3.8 Working with a triangulation consisting of rectangles, an interpolation operator $I_{h}^{k}: C^{0}(\Omega) \rightarrow S_{h}^{0}$ constructed in [16, p. 108] satisfies

$$
\sup _{v_{h} \in S_{h}} \frac{\int_{\Omega} \nabla\left(w-I_{h}^{k} w\right) \cdot \nabla v_{h} d x}{\left\|v_{h}\right\|_{0, \Omega}} \leq C h^{k+1}\|w\|_{k+3, \Omega},
$$

where $C>0$ and $v_{h}$ or $w=\frac{\partial w}{\partial \boldsymbol{n}}=0$ on $\partial \Omega$, see [23, Lemmas 1 and 2]. Using this interpolation operator, the error estimate in Theorem 3.7 can be improved to

$$
\left\|\left(u-u_{h}, \phi-\phi_{h}\right)\right\|_{\boldsymbol{V}} \leq C h^{k}\left(\|u\|_{k+3, \Omega}+|p|_{k, \Omega}\right)
$$

under additional regularity assumption on the stream function $u$.

\subsection{Construction of Space $M_{h}$}

Our goal is to obtain an efficient numerical scheme in which all the auxiliary variables except the stream function can be statically condensed out from the system. The other variables can be obtained in a post-processing step after computing the stream function. To this end, we 
want to have the mass matrix corresponding to the bilinear form $\int_{\Omega} \mu_{h} q_{h} d x$ for $\mu_{h} \in M_{h}$ and $q_{h} \in Q_{h}$ to be a diagonal matrix.

Let $\left\{\varphi_{1}, \ldots, \varphi_{n}\right\}$ be the finite element basis of the space $Q_{h}$. The finite element basis $\left\{\mu_{1}, \ldots, \mu_{n}\right\}$ of the space $M_{h}$ with $\operatorname{supp} \mu_{i}=\operatorname{supp} \varphi_{i}, 1 \leq i \leq n$, are constructed in such a way that the basis functions of $Q_{h}$ and $M_{h}$ satisfy a condition of biorthogonality relation

$$
\int_{\Omega} \mu_{i} \varphi_{j} d x=c_{j} \delta_{i j}, \quad c_{j} \neq 0,1 \leq i, j \leq n
$$

where $n:=\operatorname{dim} M_{h}=\operatorname{dim} Q_{h}, \delta_{i j}$ is the Kronecker symbol, and $c_{j}$ a scaling factor. This scaling factor $c_{j}$ can be chosen as proportional to the area $\left|\operatorname{supp} \phi_{j}\right|$.

Construction of local basis functions of the space $M_{h}$ satisfying conditions (3.1), (3.2) and (3.19) for different finite element spaces can be found in [19, 20, 22, 32]. Working with nodal finite element basis functions based on Gauss-Lobatto quadrature nodes for rectangular or hexahedral triangulation, we have shown the construction of local basis functions of $M_{h}$ satisfying conditions (3.19), (3.1) and (3.2) for an arbitrary order finite element space [22]. The construction of local basis functions of the space $M_{h}$ satisfying all the above assumptions for the lowest order simplicial finite element space can be found in [18]. Unfortunately, the construction of the local basis functions of the space $M_{h}$ with all these properties for higher order non-tensor product finite element spaces is not possible. In particular, it is shown in [19] that it is not possible to construct $M_{h}$ whose basis functions have the same support as the basis functions of $Q_{h}$ satisfying above conditions for the quadratic simplicial and serendipity elements. In order to deal with these cases, we relax the biorthogonality condition to a quasi-biorthogonal one in [20,21], where the basis functions of the space $M_{h}$ are chosen in such a way that the global mass matrix $\boldsymbol{D}$ associated with the bilinear form $\int_{\Omega} \mu_{h} q_{h} d x$ for $q_{h} \in Q_{h}$ and $\mu_{h} \in M_{h}$ is of the form

$$
\boldsymbol{D}=\left[\begin{array}{cc}
\boldsymbol{D}_{1} & 0 \\
\boldsymbol{R} & \boldsymbol{D}_{2}
\end{array}\right],
$$

where $\boldsymbol{D}_{1}$ and $\boldsymbol{D}_{2}$ are diagonal matrices and $\boldsymbol{R}$ is a rectangular matrix. The matrix $\boldsymbol{D}$ is then easy to invert and the inverse remains sparse. A matrix having this structure will be called a quasi-diagonal matrix. Under this relaxed notion, the local basis functions of the space $M_{h}$ can be constructed for the quadratic simplicial and quadratic serendipity elements as in [20] by modifying the finite element basis functions corresponding to the vertices. Since the condition $\operatorname{dim} M_{h}=\operatorname{dim} Q_{h}$ was not fulfilled in the construction of these basis functions in $[19,20]$, we provide the construction of local basis functions satisfying the assumption $\operatorname{dim} M_{h}=\operatorname{dim} Q_{h}$ for these cases.

\subsubsection{Quadratic Serendipity Elements}

Assume that $\hat{\varphi}_{i}^{l}, 1 \leq i \leq 4$, and $\hat{\varphi}_{i}^{q}, 1 \leq i \leq 8$, be the bilinear and quadratic serendipity finite element basis functions on the reference square $\hat{T}:=(-1,1) \times(-1,1)$, respectively. Here $\hat{\varphi}_{i}^{q}, 1 \leq i \leq 4$, are the basis functions corresponding to the vertices of $\hat{T}$, and $\hat{\varphi}_{i}^{q}, 5 \leq i \leq 8$, are the ones corresponding to the edges of $\hat{T}$, see the right picture of Fig. 1. For some $\alpha \neq 1$, we set

$$
\begin{aligned}
& \hat{\mu}_{1}^{q}:=\frac{\hat{\varphi}_{1}^{l}+\alpha\left(\hat{\varphi}_{3}^{l}-\hat{\varphi}_{2}^{l}-\hat{\varphi}_{4}^{l}\right)}{1-\alpha}, \quad \hat{\mu}_{2}^{q}:=\frac{\hat{\varphi}_{2}^{l}+\alpha\left(\hat{\varphi}_{4}^{l}-\hat{\varphi}_{1}^{l}-\hat{\varphi}_{3}^{l}\right)}{1-\alpha}, \\
& \hat{\mu}_{3}^{q}:=\frac{\hat{\varphi}_{3}^{l}+\alpha\left(\hat{\varphi}_{1}^{l}-\hat{\varphi}_{2}^{l}-\hat{\varphi}_{4}^{l}\right)}{1-\alpha}, \quad \text { and } \quad \hat{\mu}_{4}^{q}:=\frac{\hat{\varphi}_{4}^{l}+\alpha\left(\hat{\varphi}_{2}^{l}-\hat{\varphi}_{1}^{l}-\hat{\varphi}_{3}^{l}\right)}{1-\alpha} .
\end{aligned}
$$


Fig. 1 Ordering of the local finite element basis functions, quadratic simplicial (left) and quadratic serendipity (right)
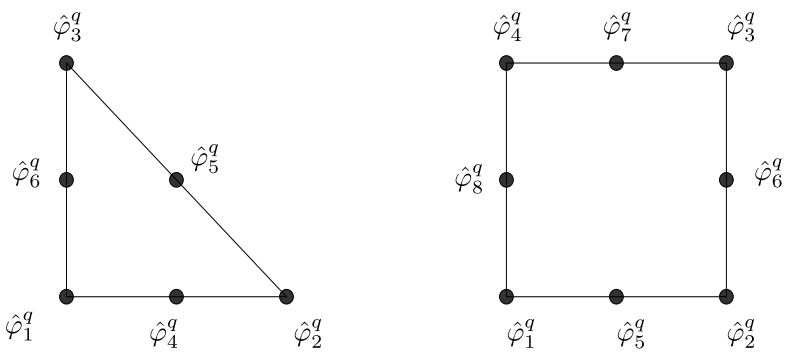

Now, defining $\tilde{\varphi}_{1}^{q}:=\hat{\varphi}_{1}^{q}-\theta\left(\hat{\varphi}_{5}^{q}+\hat{\varphi}_{8}^{q}\right), \tilde{\varphi}_{2}^{q}:=\hat{\varphi}_{2}^{q}-\theta\left(\hat{\varphi}_{5}^{q}+\hat{\varphi}_{6}^{q}\right), \tilde{\varphi}_{3}^{q}:=\hat{\varphi}_{3}^{q}-\theta\left(\hat{\varphi}_{6}^{q}+\hat{\varphi}_{7}^{q}\right)$, and $\tilde{\varphi}_{4}^{q}:=\hat{\varphi}_{4}^{q}-\theta\left(\hat{\varphi}_{7}^{q}+\hat{\varphi}_{8}^{q}\right)$, we find that with $\alpha=\frac{1}{6}$ and $\theta=-\frac{1}{5}$,

$$
\left[\left\langle\tilde{\varphi}_{i}^{q}, \hat{\mu}_{j}^{q}\right\rangle_{0, \hat{T}}\right]_{1 \leq i, j \leq 4}=\frac{1}{5} I_{4},
$$

where $I_{4}$ is the identity matrix of size $4 \times 4$. The modified finite element basis functions on vertices $\tilde{\varphi}_{i}^{q}, 1 \leq i \leq 4$, vanish on the opposite edges and are symmetric in the barycentric coordinates. Since the global basis functions obtained by mapping the modified local ones $\hat{\mu}_{i}^{q}, 1 \leq i \leq 4$, with some affine mapping and gluing them together will not be continuous, they do not span the space of all continuous piecewise bilinear functions with respect to the partition $\mathcal{T}_{h}$ on $\Omega$. However, since $\hat{\mu}_{1}^{q}+\hat{\mu}_{2}^{q}=\hat{\varphi}_{1}^{l}+\hat{\varphi}_{2}^{l}, \hat{\mu}_{2}^{q}+\hat{\mu}_{3}^{q}=\hat{\varphi}_{2}^{l}+\hat{\varphi}_{3}^{l}, \hat{\mu}_{3}^{q}+\hat{\mu}_{4}^{q}=\hat{\varphi}_{3}^{l}+\hat{\varphi}_{4}^{l}$, and $\hat{\mu}_{4}^{q}+\hat{\mu}_{1}^{q}=\hat{\varphi}_{4}^{l}+\hat{\varphi}_{1}^{l}$, we find $\hat{\mu}_{1}^{q}+\hat{\mu}_{2}^{q}=\frac{1-y}{2}, \hat{\mu}_{2}^{q}+\hat{\mu}_{3}^{q}=\frac{1+x}{2}, \hat{\mu}_{3}^{q}+\hat{\mu}_{4}^{q}=\frac{1+y}{2}$, and $\hat{\mu}_{4}^{q}+\hat{\mu}_{1}^{q}=\frac{1-x}{2}$. Denoting the edge joining the corners $i$ and $j$ of the reference element $\hat{T}$ by $e_{i j}$ for four edges $\left(e_{12}, e_{23}, e_{34}\right.$, and $\left.e_{41}\right)$, we see that $\hat{\mu}_{i}^{q}+\hat{\mu}_{j}^{q}=1$ on $e_{i j}$, and $\hat{\mu}_{i}^{q}+$ $\hat{\mu}_{j}^{q}=0$ on the edge opposite to $e_{i j}$. Thus we can conclude that the global basis functions obtained by mapping the modified local ones with some affine mapping and gluing them together will reproduce any continuous piecewise linear polynomial. Since the four local basis functions $\hat{\mu}_{i}^{q}, 1 \leq i \leq 4$, corresponding to the vertices of the reference square has the required approximation property, other four local basis functions corresponding to the four edges can be taken to be four quadratic functions which are biorthogonal to the four local basis functions $\tilde{\varphi}_{i}^{q}, 5 \leq i \leq 8$. These four local basis functions are given by

$$
\begin{aligned}
& \hat{\mu}_{5}^{q}(x, y)=-\frac{63}{5}+108 x+\frac{27}{5} y-108 x^{2}-162 x y+18 y^{2}+162 x^{2} y, \\
& \hat{\mu}_{6}^{q}(x, y)=\frac{54}{5}-\frac{207}{5} x-54 y+18 x^{2}+162 x y+54 y^{2}-162 x y^{2}, \\
& \hat{\mu}_{7}^{q}(x, y)=\frac{54}{5}-54 x-\frac{207}{5} y+54 x^{2}+162 x y+18 y^{2}-162 x^{2} y, \quad \text { and } \\
& \hat{\mu}_{8}^{q}(x, y)=-\frac{63}{5}+\frac{27}{5} x+108 y+18 x^{2}-162 x y-108 y^{2}+162 x y^{2} .
\end{aligned}
$$

\subsubsection{Simplicial Quadratic Finite Elements}

Following the same idea as in the previous paragraph, we show the construction of local basis functions of $M_{h}$ for the simplicial quadratic finite elements, see also [20]. Assume that $\hat{\varphi}_{i}^{l}, 1 \leq i \leq 3$, and $\hat{\varphi}_{i}^{q}, 1 \leq i \leq 6$, be the linear and quadratic finite element basis functions on the reference triangle $\hat{T}$, respectively. Here $\hat{\varphi}_{i}^{q}, 1 \leq i \leq 3$, are the basis functions 
corresponding to the vertices of the reference triangle $\hat{T}$ and $\hat{\varphi}_{i}^{q}, 4 \leq i \leq 6$, are the ones corresponding to the edges of $\hat{T}$, where $\hat{T}:=\{(x, y) \mid 0 \leq x, 0 \leq y, x+y \leq 1\}$, see the left picture of Fig. 1. Letting $\hat{\mu}_{i}^{q}=\hat{\varphi}_{i}^{l} 1 \leq i \leq 3$, we have

$$
\left[\left\langle\hat{\varphi}_{i}^{q}, \hat{\mu}_{j}^{q}\right\rangle_{0, \hat{T}}\right]_{1 \leq i, j \leq 3}=\left[\begin{array}{ccc}
\frac{1}{60} & -\frac{1}{120} & -\frac{1}{120} \\
-\frac{1}{120} & \frac{1}{60} & -\frac{1}{120} \\
-\frac{1}{120} & -\frac{1}{120} & \frac{1}{60}
\end{array}\right] .
$$

Hence if we define

$\tilde{\varphi}_{1}^{q}:=\hat{\varphi}_{1}^{q}+\frac{1}{12}\left(\hat{\varphi}_{4}^{q}+\hat{\varphi}_{6}^{q}\right), \quad \tilde{\varphi}_{2}^{q}:=\hat{\varphi}_{2}^{q}+\frac{1}{12}\left(\hat{\varphi}_{4}^{q}+\hat{\varphi}_{5}^{q}\right), \quad$ and $\quad \tilde{\varphi}_{3}^{q}:=\hat{\varphi}_{3}^{q}+\frac{1}{12}\left(\hat{\varphi}_{5}^{q}+\hat{\varphi}_{6}^{q}\right)$,

we obtain

$$
\left[\left\langle\tilde{\varphi}_{i}^{q}, \hat{\mu}_{j}^{q}\right\rangle_{0, \hat{T}}\right]_{1 \leq i, j \leq 3}=\frac{1}{36} I_{3}
$$

where $I_{3}$ is the identity matrix of size $3 \times 3$. We point out that the modified vertex basis functions $\tilde{\varphi}_{i}^{q}, 1 \leq i \leq 3$, vanish on the opposite edges and are symmetric in the barycentric coordinates as in the previous paragraph. As the three local basis functions corresponding to the vertices of the reference triangle are sufficient for the approximation purpose, the other three local basis functions corresponding to the three edges can be defined just to fulfill the quasi-biorthogonality property. These three basis functions are then given by

$$
\begin{aligned}
& \hat{\mu}_{4}^{q}(x, y)=-10+120 x-10 y-120 x^{2}-120 x y+30 y^{2}, \\
& \hat{\mu}_{5}^{q}(x, y)=10-50 x-50 y+30 x^{2}+180 x y+30 y^{2}, \quad \text { and } \\
& \hat{\mu}_{6}^{q}(x, y)=-10-10 x+120 y+30 x^{2}-120 x y-120 y^{2} .
\end{aligned}
$$

If we use the ordering of the local basis functions as shown in Fig. 1, the local matrix associated with the bilinear form $\int_{\Omega} \mu_{h} q_{h} d x$ for $q_{h} \in Q_{h}$ and $\mu_{h} \in M_{h}$ is exactly of the form (3.20). Using the global ordering in such a way that the degree of freedom corresponding to edges come after the degree of freedom corresponding to vertices, the global matrix $\boldsymbol{D}$ will have the structure as in (3.20).

Remark 3.9 In order to show that our basis functions for $M_{h}$ and $Q_{h}$ satisfy inequality (3.1), we proceed as follows. Let $h_{i}$ be the local mesh-size at $i$-th node, and let

$$
q_{h}=\sum_{i=1}^{n} a_{i} \varphi_{i}, \quad \mu_{h}=\sum_{i=1}^{n} a_{i} \mu_{i} .
$$

Then by using the structure of the Gram matrix, we can show that

$$
\int_{\Omega} q_{h} \mu_{h} d x
$$

$\left\|q_{h}\right\|_{0, \Omega}^{2}$ and $\left\|\mu_{h}\right\|_{0, \Omega}^{2}$ are equivalent to $\sum_{i=1}^{n_{k}} h_{i}^{2} a_{i}^{2}$, which guarantees (3.1), see also [19].

Remark 3.10 We have assumed $\operatorname{dim} M_{h}=\operatorname{dim} Q_{h}$ for both clamped and simply supported boundary conditions. In the case of clamped boundary condition, this condition is automatically satisfied as $\operatorname{dim} M_{h}=\operatorname{dim} Q_{h}$ by construction. However, for the simply supported 
boundary condition, the degree of freedom of $M_{h}$ corresponding the Dirichlet nodes should be removed to satisfy the condition $\operatorname{dim} M_{h}=\operatorname{dim} Q_{h}$. The removal of the degree of freedom of $M_{h}$ corresponding to the boundary of the interface is called boundary modification in the context of mortar finite elements, which is essential to obtain optimal a priori estimates [19, $20,22]$. We note that the same modification as discussed in $[19,20,22]$ should be applied to the basis functions of $M_{h}$ so that the approximation property of $M_{h}$ remains valid even after removing the degree of freedom corresponding to the Dirichlet nodes.

\subsection{Algebraic Formulation}

To arrive at the algebraic formulation, we consider the discrete saddle point problem (3.4) in more detail. Choosing the test function $v_{h}=0$ and $\psi_{h}=0$ successively in the first equation of (3.4), we have

$$
\begin{aligned}
\int_{\Omega} \phi_{h} \psi_{h} d x+\int_{\Omega} p_{h} \psi_{h} d x & =0, \quad \psi_{h} \in M_{h}, \\
\int_{\Omega} \nabla v_{h} \cdot \nabla p_{h} d x & =\ell\left(v_{h}\right), \quad v_{h} \in S_{h}^{0} .
\end{aligned}
$$

In the following, we use the same notation for the vector representation of the solution and the solution as elements in $\boldsymbol{V}_{h}$ and $Q_{h}$. Let $\boldsymbol{A}, \boldsymbol{M}$ and $\boldsymbol{D}$ be the matrices associated with the bilinear forms $\int_{\Omega} \nabla v_{h} \cdot \nabla q_{h} d x, \int_{\Omega} \phi_{h} \psi_{h} d x$ and $\int_{\Omega} \phi_{h} q_{h} d x$, respectively, where $v_{h} \in S_{h}^{0}, q_{h} \in Q_{h}, \phi_{h} \in M_{h}$ and $\psi_{h} \in M_{h}$. Then the algebraic formulation of the saddle point problem (3.4) is given by

$$
\left[\begin{array}{ccc}
0 & 0 & \boldsymbol{A}^{T} \\
0 & \boldsymbol{M} & \boldsymbol{D}^{T} \\
\boldsymbol{A} & \boldsymbol{D} & 0
\end{array}\right]\left[\begin{array}{c}
u_{h} \\
\phi_{h} \\
p_{h}
\end{array}\right]=\left[\begin{array}{c}
f_{h} \\
0 \\
0
\end{array}\right],
$$

where $f_{h}$ is the discretized vector associated with the linear form $\ell\left(v_{h}\right)$. Since the matrix $\boldsymbol{D}$ is diagonal or quasi-diagonal, we can do the static condensation of unknowns $\phi_{h}$ and $p_{h}$, and arrive at the following linear system based on unknown associated only with the stream function:

$$
\left(\boldsymbol{A}^{T}\left(\boldsymbol{D}^{-1}\right)^{T} \boldsymbol{M} \boldsymbol{D}^{-1} \boldsymbol{A}\right) u_{h}=f_{h} .
$$

The vorticity $\phi_{h}$ and the Lagrange multiplier $p_{h}$ can be computed after solving (3.22) by simply inverting a diagonal or quasi-diagonal matrix using the second and third blocks of (3.21).

Remark 3.11 Because of the special structure of inverse of the matrix $\boldsymbol{D}$, the system matrix in (3.22) is sparse. It is important to have the system matrix to have sparse structure if an iterative solver is to be applied. However, the condition number of the system matrix in (3.22) is worse than a system matrix arising from the discretization Poisson equation, see [2, 7]. The condition number of our system is the same as the original approach proposed by Ciarlet and Raviart [10], see also [2, 25, 29]. Therefore, one has to apply similar preconditioners for the system (3.22) as proposed in [2, 25, 29] to introduce an efficient iterative methods. Since we do not focus on this aspect of this approach, we simply solve the problem by using LU factorization. 
Table 1 Discretization errors in different norms for the clamped boundary condition, triangles and linear

\begin{tabular}{lrllllll}
\hline Level & \# elem. & $\frac{\left\|u-u_{h}\right\|_{0, \Omega}}{\|u\|_{0, \Omega}}$ & & $\frac{\left|u-u_{h}\right|_{1, \Omega}}{|u|_{1, \Omega}}$ & & $\frac{\left\|\phi-\phi_{h}\right\|_{0, \Omega}}{\|\phi\|_{0, \Omega}}$ \\
\hline 0 & 32 & $5.34290 \mathrm{e}-01$ & & $6.32693 \mathrm{e}-01$ & & $6.32041 \mathrm{e}-01$ \\
1 & 128 & $3.26972 \mathrm{e}-01$ & 0.71 & $4.01635 \mathrm{e}-01$ & 0.66 & $5.16879 \mathrm{e}-01$ & 0.29 \\
2 & 512 & $1.30302 \mathrm{e}-01$ & 1.33 & $1.89139 \mathrm{e}-01$ & 1.09 & $3.34937 \mathrm{e}-01$ & 0.63 \\
3 & 2048 & $3.99107 \mathrm{e}-02$ & 1.71 & $8.32646 \mathrm{e}-02$ & 1.18 & $1.88319 \mathrm{e}-01$ & 0.83 \\
4 & 8192 & $1.08809 \mathrm{e}-02$ & 1.87 & $3.88438 \mathrm{e}-02$ & 1.10 & $9.92016 \mathrm{e}-02$ & 0.93 \\
5 & 32768 & $2.82773 \mathrm{e}-03$ & 1.94 & $1.89646 \mathrm{e}-02$ & 1.03 & $5.08074 \mathrm{e}-02$ & 0.97 \\
6 & 131072 & $7.19891 \mathrm{e}-04$ & 1.97 & $9.41839 \mathrm{e}-03$ & 1.01 & $2.56967 \mathrm{e}-02$ & 0.98 \\
7 & 524288 & $1.81559 \mathrm{e}-04$ & 1.99 & $4.70081 \mathrm{e}-03$ & 1.00 & $1.29204 \mathrm{e}-02$ & 0.99 \\
\hline
\end{tabular}

\section{Numerical Results}

In this section, we show some numerical experiments for the biharmonic equation using clamped and simply supported boundary conditions. In particular, we want to compute the convergence rates of the relative errors in $L^{2}$ - and $H^{1}$-norms for the stream function and the convergence rates of the relative errors in $L^{2}$-norm for the vorticity using linear and quadratic finite element spaces.

In all our examples we solve $\Delta^{2} u=f$ using the clamped and simply supported boundary condition in $\Omega:=(0,1)^{2}$, where the right hand side $f$ is computed by using the exact solution.

In our first example, we consider the exact solution

$$
u(x, y)=\left(e^{x}+(x+1) e^{y}\right) x^{2} y^{2}(1-x)^{2}(1-y)^{2},
$$

which satisfies the clamped boundary condition with $u=0$ and $\frac{\partial u}{\partial \boldsymbol{n}}=0$ on $\partial \Omega$. Discretization errors along with the rates of convergence in various steps of refinement and in various norms are given in Tables 1-5. In all our tables, we observe the optimal asymptotic convergence rates as predicted by the theory. As can be seen from these tables, the relative $L^{2}$-error in vorticity shows the convergence rate of order $h$ in the linear and bilinear case for simplicial and rectangular partition, the quadratic case shows a different picture. For the simplicial partition, the convergence rate is only of order $h^{\frac{3}{2}}$, whereas the convergence rate is of order $h^{2}$ for the rectangular partition using serendipity elements showing a better convergence behavior in case of rectangular partition of $\Omega$. The convergence rates for the vorticity are even better for biquadratic finite elements. We note that the space $M_{h}$ for the quadratic serendipity case contains only linear finite element space, whereas the space $M_{h}$ for the biquadratic case contains bilinear finite element space.

Remark 4.1 We have a sharp convergence rate for the quadratic simplicial elements in contrast to better convergence rates for other cases. Although we obtain theoretically predicted convergence rates all cases, the better convergence rates in some cases give a hope to improve the theoretical convergence rates in these cases. The better convergence for the quadrilateral case is explained by using the theory in [23]. However, more investigation is necessary in the simplicial cases. 
Table 2 Discretization errors in different norms for the clamped boundary condition, rectangles and bilinear

\begin{tabular}{lrllllll}
\hline Level & \# elem. & $\frac{\left\|u-u_{h}\right\|_{0, \Omega}}{\|u\|_{0, \Omega}}$ & & $\frac{\left|u-u_{h}\right|_{1, \Omega}}{|u|_{1, \Omega}}$ & & $\frac{\left\|\phi-\phi_{h}\right\|_{0, \Omega}}{\|\phi\|_{0, \Omega}}$ \\
\hline 0 & 16 & $3.63341 \mathrm{e}-01$ & 0 & $5.05331 \mathrm{e}-01$ & 0 & $5.77392 \mathrm{e}-01$ & 0 \\
1 & 64 & $2.18880 \mathrm{e}-01$ & 0.73 & $3.06596 \mathrm{e}-01$ & 0.72 & $4.61843 \mathrm{e}-01$ & 0.32 \\
2 & 256 & $8.02639 \mathrm{e}-02$ & 1.45 & $1.40782 \mathrm{e}-01$ & 1.12 & $2.84812 \mathrm{e}-01$ & 0.70 \\
3 & 1024 & $2.36341 \mathrm{e}-02$ & 1.76 & $6.40446 \mathrm{e}-02$ & 1.14 & $1.56135 \mathrm{e}-01$ & 0.87 \\
4 & 4096 & $6.36319 \mathrm{e}-03$ & 1.89 & $3.07178 \mathrm{e}-02$ & 1.06 & $8.14364 \mathrm{e}-02$ & 0.94 \\
5 & 16384 & $1.64784 \mathrm{e}-03$ & 1.95 & $1.51580 \mathrm{e}-02$ & 1.02 & $4.15487 \mathrm{e}-02$ & 0.97 \\
6 & 65536 & $4.19102 \mathrm{e}-04$ & 1.98 & $7.55147 \mathrm{e}-03$ & 1.01 & $2.09804 \mathrm{e}-02$ & 0.99 \\
7 & 262144 & $1.05669 \mathrm{e}-04$ & 1.99 & $3.77214 \mathrm{e}-03$ & 1.00 & $1.05415 \mathrm{e}-02$ & 0.99 \\
\hline
\end{tabular}

Table 3 Discretization errors in different norms for the clamped boundary condition, triangles and quadratic

\begin{tabular}{lrllllll}
\hline Level & \# elem. & $\frac{\left\|u-u_{h}\right\|_{0, \Omega}}{\|u\|_{0, \Omega}}$ & & $\frac{\left|u-u_{h}\right|_{1, \Omega}}{|u|_{1, \Omega}}$ & & $\frac{\left\|\phi-\phi_{h}\right\|_{0, \Omega}}{\|\phi\|_{0, \Omega}}$ \\
\hline 0 & 32 & $7.21130 \mathrm{e}-02$ & & $1.73764 \mathrm{e}-01$ & & $2.24893 \mathrm{e}-01$ \\
1 & 128 & $7.67699 \mathrm{e}-03$ & 3.23 & $4.61574 \mathrm{e}-02$ & 1.91 & $7.39127 \mathrm{e}-02$ & 1.61 \\
2 & 512 & $8.36813 \mathrm{e}-04$ & 3.20 & $1.18158 \mathrm{e}-02$ & 1.97 & $2.48864 \mathrm{e}-02$ & 1.57 \\
3 & 2048 & $1.00174 \mathrm{e}-04$ & 3.06 & $2.97468 \mathrm{e}-03$ & 1.99 & $8.77516 \mathrm{e}-03$ & 1.50 \\
4 & 8192 & $1.24057 \mathrm{e}-05$ & 3.01 & $7.44945 \mathrm{e}-04$ & 2 & $3.12711 \mathrm{e}-03$ & 1.49 \\
5 & 32768 & $1.54909 \mathrm{e}-06$ & 3 & $1.86302 \mathrm{e}-04$ & 2 & $1.11290 \mathrm{e}-03$ & 1.49 \\
6 & 131072 & $1.93720 \mathrm{e}-07$ & 3 & $4.65777 \mathrm{e}-05$ & 2 & $3.95040 \mathrm{e}-04$ & 1.49 \\
\hline
\end{tabular}

Table 4 Discretization errors in different norms for the clamped boundary condition, rectangles and quadratic serendipity

\begin{tabular}{lrllllll}
\hline Level & \# elem. & \multicolumn{1}{l}{$\frac{\left\|u-u_{h}\right\|_{0, \Omega}}{\|u\|_{0, \Omega}}$} & & $\frac{\left|u-u_{h}\right|_{1, \Omega}}{|u|_{1, \Omega}}$ & & $\frac{\left\|\phi-\phi_{h}\right\|_{0, \Omega}}{\|\phi\|_{0, \Omega}}$ \\
\hline 0 & 16 & $3.51301 \mathrm{e}-02$ & & $1.36042 \mathrm{e}-01$ & & $1.57615 \mathrm{e}-01$ \\
1 & 64 & $3.71421 \mathrm{e}-03$ & 3.24 & $3.38321 \mathrm{e}-02$ & 2.01 & $4.40283 \mathrm{e}-02$ & 1.84 \\
2 & 256 & $4.20670 \mathrm{e}-04$ & 3.14 & $8.46059 \mathrm{e}-03$ & 2 & $1.09815 \mathrm{e}-02$ & 2.00 \\
3 & 1024 & $5.08994 \mathrm{e}-05$ & 3.05 & $2.11683 \mathrm{e}-03$ & 2 & $2.57099 \mathrm{e}-03$ & 2.09 \\
4 & 4096 & $6.32166 \mathrm{e}-06$ & 3.01 & $5.29376 \mathrm{e}-04$ & 2 & $6.05036 \mathrm{e}-04$ & 2.09 \\
5 & 16384 & $7.89256 \mathrm{e}-07$ & 3 & $1.32357 \mathrm{e}-04$ & 2 & $1.45262 \mathrm{e}-04$ & 2.06 \\
6 & 65536 & $9.86327 \mathrm{e}-08$ & 3 & $3.30902 \mathrm{e}-05$ & 2 & $3.54722 \mathrm{e}-05$ & 2.03 \\
\hline
\end{tabular}

Our second example is concerned with the exact solution $u$ defined by

$$
u(x, y)=\left(y e^{x}+x e^{y}\right) x^{3} y^{3}(1-x)^{3}(1-y)^{3},
$$

which satisfies the simply supported boundary condition $u=0$ and $\Delta u=0$ on $\partial \Omega$. We compute the relative errors in $L^{2}$ and $H^{1}$-norms for the stream function and the relative errors in $L^{2}$-norm for the vorticity using linear and bilinear finite element space and the corresponding space $M_{h}$. As can be seen in Tables 6-7, the convergence rates for the stream function are of order $h$ and $h^{2}$ in $H^{1}$ and $L^{2}$-norms, respectively, and the convergence rate 
Table 5 Discretization errors in different norms for the clamped boundary condition, rectangles and biquadratic

\begin{tabular}{lrllllll}
\hline Level & \# elem. & $\frac{\left\|u-u_{h}\right\|_{0, \Omega}}{\|u\|_{0, \Omega}}$ & & $\frac{\left|u-u_{h}\right|_{1, \Omega}}{|u|_{1, \Omega}}$ & & $\frac{\left\|\phi-\phi_{h}\right\|_{0, \Omega}}{\|\phi\|_{0, \Omega}}$ \\
\hline 0 & 16 & $2.35528 \mathrm{e}-02$ & & $1.27617 \mathrm{e}-01$ & & $2.46599 \mathrm{e}-02$ & \\
1 & 64 & $3.15699 \mathrm{e}-03$ & 2.90 & $3.33867 \mathrm{e}-02$ & 1.93 & $3.23521 \mathrm{e}-03$ & 2.93 \\
2 & 256 & $4.01609 \mathrm{e}-04$ & 2.97 & $8.43997 \mathrm{e}-03$ & 1.98 & $4.14979 \mathrm{e}-04$ & 2.96 \\
3 & 1024 & $5.04224 \mathrm{e}-05$ & 2.99 & $2.11584 \mathrm{e}-03$ & 2 & $5.28399 \mathrm{e}-05$ & 2.97 \\
4 & 4096 & $6.30974 \mathrm{e}-06$ & 3 & $5.29325 \mathrm{e}-04$ & 2 & $6.68037 \mathrm{e}-06$ & 2.98 \\
5 & 16384 & $7.88934 \mathrm{e}-07$ & 3 & $1.32354 \mathrm{e}-04$ & 2 & $8.40308 \mathrm{e}-07$ & 2.99 \\
\hline
\end{tabular}

Table 6 Discretization errors in different norms for the simply supported boundary condition, triangles and linear

\begin{tabular}{lrllllll}
\hline Level & \# elem. & $\frac{\left\|u-u_{h}\right\|_{0, \Omega}}{\|u\|_{0, \Omega}}$ & & $\frac{\left|u-u_{h}\right|_{1, \Omega}}{|u|_{1, \Omega}}$ & & \multicolumn{2}{c}{$\frac{\left\|\phi-\phi_{h}\right\|_{0, \Omega}}{\|\phi\|_{0, \Omega}}$} \\
\hline 0 & 32 & $1.00000 \mathrm{e}+00$ & & $1.00000 \mathrm{e}+00$ & & $1.00000 \mathrm{e}+00$ \\
1 & 128 & $7.12820 \mathrm{e}-01$ & 0.49 & $7.14584 \mathrm{e}-01$ & 0.49 & $7.83438 \mathrm{e}-01$ & 0.350 \\
2 & 512 & $2.10740 \mathrm{e}-01$ & 1.76 & $2.57361 \mathrm{e}-01$ & 1.47 & $4.16608 \mathrm{e}-01$ & 0.911 \\
3 & 2048 & $5.80843 \mathrm{e}-02$ & 1.86 & $1.00223 \mathrm{e}-01$ & 1.36 & $2.23862 \mathrm{e}-01$ & 0.896 \\
4 & 8192 & $1.60359 \mathrm{e}-02$ & 1.86 & $4.40289 \mathrm{e}-02$ & 1.19 & $1.16898 \mathrm{e}-01$ & 0.937 \\
5 & 32768 & $4.20144 \mathrm{e}-03$ & 1.93 & $2.09303 \mathrm{e}-02$ & 1.07 & $5.92290 \mathrm{e}-02$ & 0.981 \\
6 & 131072 & $1.07363 \mathrm{e}-03$ & 1.97 & $1.03099 \mathrm{e}-02$ & 1.02 & $2.97219 \mathrm{e}-02$ & 0.995 \\
7 & 524288 & $2.71255 \mathrm{e}-04$ & 1.98 & $5.13455 \mathrm{e}-03$ & 1.01 & $1.48750 \mathrm{e}-02$ & 0.999 \\
\hline
\end{tabular}

Table 7 Discretization errors in different norms for the simply supported boundary condition, rectangles and bilinear

\begin{tabular}{lrllllll}
\hline Level & \# elem. & $\frac{\left\|u-u_{h}\right\|_{0, \Omega}}{\|u\|_{0, \Omega}}$ & & $\frac{\left|u-u_{h}\right|_{1, \Omega}}{|u|_{1, \Omega}}$ & & $\frac{\left\|\phi-\phi_{h}\right\|_{0, \Omega}}{\|\phi\|_{0, \Omega}}$ \\
\hline 0 & 16 & $1.00000 \mathrm{e}+00$ & & $1.00000 \mathrm{e}+00$ & & $1.00000 \mathrm{e}+00$ \\
1 & 64 & $8.59993 \mathrm{e}-01$ & 0.22 & $8.42251 \mathrm{e}-01$ & 0.25 & $8.91205 \mathrm{e}-01$ & 0.17 \\
2 & 256 & $1.43223 \mathrm{e}-01$ & 2.59 & $1.95467 \mathrm{e}-01$ & 2.11 & $3.67212 \mathrm{e}-01$ & 1.28 \\
3 & 1024 & $2.46450 \mathrm{e}-02$ & 2.54 & $7.05286 \mathrm{e}-02$ & 1.47 & $1.83978 \mathrm{e}-01$ & 1.00 \\
4 & 4096 & $6.17898 \mathrm{e}-03$ & 2 & $3.25399 \mathrm{e}-02$ & 1.12 & $9.54341 \mathrm{e}-02$ & 0.95 \\
5 & 16384 & $1.55362 \mathrm{e}-03$ & 2 & $1.58651 \mathrm{e}-02$ & 1.04 & $4.82796 \mathrm{e}-02$ & 0.98 \\
6 & 65536 & $3.88985 \mathrm{e}-04$ & 2 & $7.87881 \mathrm{e}-03$ & 1.01 & $2.42181 \mathrm{e}-02$ & 1.00 \\
7 & 262144 & $9.72822 \mathrm{e}-05$ & 2 & $3.93257 \mathrm{e}-03$ & 1 & $1.21194 \mathrm{e}-02$ & 1.00 \\
\hline
\end{tabular}

for the vorticity is of order $h$. We point out that the convergence rates for the vorticity can be improved by projecting the solution on the finite element space $S_{h}^{0}$ or $S_{h}$.

\section{Conclusion}

We have presented a mixed finite element method for the biharmonic equation with simply supported or clamped boundary condition using different finite element spaces for the 
stream function and vorticity. As the sets of basis functions for the discretization of the stream function and vorticity are constructed in such a way that they satisfy a condition of biorthogonality or quasi-biorthogonality, other auxiliary variables except the degree of freedom corresponding to the stream function can be statically condensed out from the system. The presented numerical results demonstrate the efficiency and optimality of the approach.

\section{References}

1. Adams, R.: Sobolev Spaces. Academic Press, New York (1975)

2. Axelsson, O., Gustafsson, I.: An iterative solver for a mixed variable variational formulation of the (first) biharmonic problem. Comput. Methods Appl. Mech. Eng. 20, 9-16 (1997)

3. Babuška, I., Osborn, J., Pitkäranta, J.: Analysis of mixed methods using mesh dependent norms. Math. Comput. 35, 1039-1062 (1980)

4. Bernardi, C., Maday, Y., Patera, A.: A new nonconforming approach to domain decomposition: the mortar element method. In: H. B. et al. (eds.) Nonlinear Partial Differential Equations and Their Applications, pp. 13-51. Paris (1994)

5. Brenner, S., Scott, L.: The Mathematical Theory of Finite Element Methods. Springer, New York (1994)

6. Brenner, S., Sung, L.-Y.: $C^{0}$ interior penalty methods for fourth order elliptic boundary value problems on polygonal domains. J. Sci. Comput. 22-23, 83-118 (2005)

7. Brezzi, F., Fortin, M.: Mixed and Hybrid Finite Element Methods. Springer, New York (1991)

8. Ciarlet, P.: The Finite Element Method for Elliptic Problems. North Holland, Amsterdam (1978)

9. Ciarlet, P., Glowinski, R.: Dual iterative techniques for solving a finite element approximation of the biharmonic equation. Comput. Methods Appl. Mech. Eng. 5, 277-295 (1975)

10. Ciarlet, P., Raviart, P.: A mixed finite element method for the biharmonic equation. In: Boor, C.D. (ed.) Symposium on Mathematical Aspects of Finite Elements in Partial Differential Equations, pp. 125-143. Academic Press, New York (1974),

11. Davini, C., Pitacco, I.: An unconstrained mixed method for the biharmonic problem. SIAM J. Numer. Anal. 38, 820-836 (2001)

12. Engel, G., Garikipati, K., Hughes, T., Larson, M., Mazzei, L., Taylor, R.: Continuous/discontinuous finite element approximations of fourth-order elliptic problems in structural and continuum mechanics with applications to thin beams and plates, and strain gradient elasticity. Comput. Methods Appl. Mech. Eng. 191, 3669-3750 (2002)

13. Falk, R.: Approximation of the biharmonic equation by a mixed finite element method. SIAM J. Numer. Anal. 15, 556-567 (1978)

14. Falk, R., Osborn, J.: Error estimates for mixed methods. RAIRO Anal. Numér. 14, 249-277 (1980)

15. Galántai, A.: Projectors and Projection Methods. Kluwer Academic, Dordrecht (2003)

16. Girault, V., Raviart, P.-A.: Finite Element Methods for Navier-Stokes Equations. Springer, Berlin (1986)

17. Grisvard, P.: Elliptic Problems in Nonsmooth Domains. Monographs and Studies in Mathematics, vol. 24. Pitman, Boston (1985). (Advanced Publishing Program)

18. Kim, C., Lazarov, R., Pasciak, J., Vassilevski, P.: Multiplier spaces for the mortar finite element method in three dimensions. SIAM J. Numer. Anal. 39, 519-538 (2001)

19. Lamichhane, B.: Higher order mortar finite elements with dual Lagrange multiplier spaces and applications. PhD thesis, Universität Stuttgart (2006)

20. Lamichhane, B., Stevenson, R., Wohlmuth, B.: Higher order mortar finite element methods in 3D with dual Lagrange multiplier bases. Numer. Math. 102, 93-121 (2005)

21. Lamichhane, B., Wohlmuth, B.: A quasi-dual Lagrange multiplier space for serendipity mortar finite elements in 3D. Math. Model. Numer. Anal. 38, 73-92 (2004)

22. Lamichhane, B., Wohlmuth, B.: Biorthogonal bases with local support and approximation properties. Math. Comput. 76, 233-249 (2007)

23. Li, J.: Full-order convergence of a mixed finite element method for fourth-order elliptic equations. J. Math. Anal. Appl. 230, 329-349 (1999)

24. Lions, J.-L., Magenes, E.: Non-homogeneous Boundary Value Problems and Applications, Vol. I. Springer, New York (1972). Translated from the French by P. Kenneth, Die Grundlehren der mathematischen Wissenschaften, Band 181

25. Mihajlović, M., Silvester, D.: Efficient parallel solvers for the biharmonic equation. Parallel Comput. 30, 35-55 (2004)

26. Monk, P.: A mixed finite element method for the biharmonic equation. SIAM J. Numer. Anal. 24, 737749 (1987) 
27. Scholz, R.: A mixed method for 4th order problems using linear finite elements. RAIRO Anal. Numér. 12, 85-90 (1978)

28. Scott, L., Zhang, S.: Finite element interpolation of nonsmooth functions satisfying boundary conditions. Math. Comput. 54, 483-493 (1990)

29. Silvester, D., Mihajlović, M.: A black-box multigrid preconditioner for the biharmonic equation. BIT Numer. Math. 44, 151-163 (2004)

30. Szyld, D.: The many proofs of an identity on the norm of oblique projections. Numer. Algorithms $\mathbf{4 2}$, 309-323 (2006)

31. Wells, G., Kuhl, E., Garikipati, K.: A discontinuous Galerkin method for the Cahn-Hilliard equation. J. Comput. Phys. 218, 860-877 (2006)

32. Wohlmuth, B.: Discretization Methods and Iterative Solvers Based on Domain Decomposition. LNCS, vol. 17. Springer, Heidelberg (2001) 